Meta

Journal des traducteurs

Translators' Journal

\title{
L'ethnologue, le traducteur et l'écrivain
}

\section{François Laplantine}

Volume 40, numéro 3, septembre 1995

La traduction, qu'est-ce à dire? Phénoménologies de la traduction

URI : https://id.erudit.org/iderudit/003398ar

DOI : https://doi.org/10.7202/003398ar

Aller au sommaire du numéro

Éditeur(s)

Les Presses de l'Université de Montréal

ISSN

0026-0452 (imprimé)

1492-1421 (numérique)

Découvrir la revue

Citer cet article

Laplantine, F. (1995). L'ethnologue, le traducteur et l'écrivain. Meta, 40(3), 497-507. https://doi.org/10.7202/003398ar

\section{Résumé de l'article}

Grappling with the issue of ethnography caught between identity and alterity. the author sets the limits and refutes the assertions of the dominant scientific model of rationality. He argues in favour of the use of language in keeping with the nature of the phenomena under study and socio-cultural diversity, citing as an example the "writing" of Latin America. Translation is therefore considered as a model for internalizing that which originates on the outside, without removing the traces of distance and difference, for complete equivalence does not exist. Like an ethnographer, the translator is confronted with a primary referent, whereas literature, translation and ethnography are used as an experiment of limits, as ways of approaching the Other and as the methodical ahandonment of identity as sameness.
Ce document est protégé par la loi sur le droit d'auteur. L'utilisation des services d’Érudit (y compris la reproduction) est assujettie à sa politique d'utilisation que vous pouvez consulter en ligne.

https://apropos.erudit.org/fr/usagers/politique-dutilisation/ 


\title{
L'ETHNOLOGUE, LE TRADUCTEUR ET L'ECRIVAIN
}

Françots Lapl.antint:

Universite Lumiere-Lyon 2, Lyon. France

\begin{abstract}
Grappling with the issue of ethnography caught between identity and alterity, the author sets the fimits and refutes the assertions of the dominant scientific model of rationali1y. He argues in favour of the use of language in keeping with the nature of the phenomena under study and socio-cultural diversity. ciring as an example the "writing" of Latin America. Translation is therefore considered as a model for internalizing that which originates on the outside. without removing the traces of distance and difference. for complete equivalence does not exist. Like an ethnographer. the translator is confronted with a primary referent, whercas literature, translation and ethnography are used as an experiment of limiss. as ways of approaching the Other and as the methodical abandonment of identity as sameness
\end{abstract}

L'anthropologie consiste à rendre familier ce qui nous est étrange et étranger (la culture des autres) et à rendre étrange voire étranger ce qui nous est familier (notre propre culture). Elle cherche à percevoir la réalité de ce que vivent les individus qui n'appartiennent pas à notre culture par les yeux de ces individus, à essayer de comprendre ce que signifie par exemple pour eux la naissance. l'amour, la maladie, la mort, et à en rendre compte de la manière la plus précise aux hommes et aux femmes de ma société. Elle vise aussi à apprendre à s'étonner des comportements qui sont les nôtres (notre manière particulière de marcher, de manger, de nous rencontrer, de nous éviter, les mots qui sontent de notre bouche à notre insu...) du fait que nous sommes nés ici et non pas là-bas, à une époque donnée et pas à une autre. Il convient pour cela de prendre de la distance par rapport à soi, d'arriver à se regarder comme un personnage insolite appréhendé à travers le regard d'un autre. Or, ce mouvement, à vrai dire vertigineux, de va-et-vient entre la familiarité et l'étrangeté est dans l'ethnographie (ethnographie = écriture de la culture) érigé en méthode. Percevoir une culture comme un texte appelant un travail artisanal qui est celui de l'interprétation, et corrélativement considérer l'anthropologue comme un auteur, ne signifie pas que la culture à laquelle je suis confronté soit un texte, mais que cette dernière ne se donne à comprendre que dans un texte fait pour être lu et renvoyant à un ensemble d'autres textes. C'est là une opération tout à fait singulière qui suppose que nous réussissions à abandonner la notion de centre, que nous renoncions à la tentation tenace de ramener encore et encore l'inconnu au connu, que nous abolissions l'idée d'évaluation (à partir d'une langue, d'un modèle, bref d'une pensée dominante, non exclusive qui serait la mesure de toutes les autres).

Or, force est de reconnaître que cette position, souvent préconisée, a bien du mal à $s$ imposer. Pour des raisons de survie, probablement, je veux dire que nous tenons tous à notre patrie, à notre quartier, à notre maison, à nos pantoufles, et que sais-je encore, bref, à notre identité dont l'inclination naturelle (c'est-à-dire culturelle) est de se constituer et de persévérer d’une manière soustractive. Par ailleurs (mais est-ce vraiment par ailleurs ?), il y a le courant largement dominant de ce que l'on appelle la rationalité scientifique pour laquelle il n'y aurait de connaissance "vraie" que, I/ médiatisée par la distance des sondages, des statistiques, voire en ethnologie des fameux «informateurs» ; 
2/ procédant du principe de causalité : A produit B qui conduit à $C$, etc. ; 3/ débusquant de l'illusion, des fantômes et de la fiction.

Cette attitude et la démarche qui a commencé à être préconisée étant à mon sens totalement irréconciliable - la science est l'espace des désaccords et des contradictions. non celui de leur résolution (religieuse, politique, esthétique) - mieux vaut reprendre successivement les trois points précédents en précisant les voies d'une alternative.

1/ L'une des questions - en fait c'est un défi - qui nous est posée ne consiste pas à s'enquérir de ce que pensent les gens qui vivent autrement, réfléchissent autrement. parlent une langue "étrangère", mais à chercher à penser dans cette langue en l'apprenant. C'est toujours en effet par la vue. l'ouie et l'odorat, et notamment par la langue et la cuisine, que débute l'apprentissage d'une culture extérieure. De Gérondo écrivait déjà : «ll faut tâcher de pénétrer ce qu'ils pensent. et non prétendre les faire penser à notre manière.» Pour cela, le chercheur doit alors devenir acteur parmi d autres acteurs.

2/ Pour beaucoup, l'accès à la rationalité scientifique se serait historiquement constituée dans la substitution du principe de causalité à celui d'analogie. Dans le "grand livre du monde», on recherchait de la cohérence partout. Or. il est bien terminé, le temps où l'on rêvait que "ce qui est en haut est comme ce qui est en bas". Bienvenue à l'avènement de l'époque moderne où l'on ne s'en laisse plus compter, où l'on sait notamment avec certitude que c'est toujours nécessairement ce qui est en bas qui produit l'agitation parfaitement illusoire de ce qui est en haut. Voici qu'une pensée cette fois analytique et non plus synthétique, animée par le rêve (le cauchemar ?) d'en finir une fois pour toutes avec le langage confus de la métaphore, une pensée enracinée dans la logique de la preuve («et vous, où étiez-vous le 7 juillet dernier à $10 \mathrm{~h} \mathrm{30} \mathrm{?»)} \mathrm{conduit.} \mathrm{par} \mathrm{glissements}$ progressifs, la recherche du côté de l'explication causale: la quête éperdue de ce qui précède. conditionne. détermine.

Je voudrais proposer ici une méthodologie des correspondances échappant à la fois au déchaînement analogique, à la réduction à l'identique («ils se comportent comme», "cela ressemble à") et à la reconstitution génétique. Je n'ai d’ailleurs personnellement rien contre le fait qu'il puisse y avoir un sens premier, disons originel, conduisant à une signification ultérieure et ainsi de suite, mais à condition de se demander s'il n'y aurait pas aussi un sens à côté du sens, un autre sens dans le sens, donc plusieurs sens qui vont et viennent, sans nécessairement entretenir des rapports chronologiques.

3/ La mode est aux souterrains, aux grottes, aux caves, aux cachots, aux cavernes, et chacun furète selon son goût et son outillage. afin de débusquer toutes les menteries et de déterrer toutes les cachotteries que l'on aurait enfouies dans les profondeurs. Pour beaucoup, il est convenu une fois pour toutes que ce qu'il convient de faire est moins de s'imprégner des discours des autres que de chercher ce qui se dissimule derriere' ou dessous. "Dévoiler" et "décrypter" sont devenus les maîtres mots. ce qui suppose qu il y ait du cryptique quelque part, la science étant une cryptologie fondée sur de minutieuses explorations cryptographiques. Comment ne pas percevoir dans cette attitude qui part à la dé-couverte de ce qui était couvert, à la recherche des logiques "cachées», de la réalité invisible qui se dissimule sous les masques dont le chercheur, lui, va nous apprendre à nous, pauvres idiots, ce qu'ils cachent, un relent d'occultisme ? D'autant qu'il est bien sûr convenu que seul un tout petit nombre (spécialistes de l'extirpation et de l'exposition à la lumière de ce qui se trouvait dans les souterrains) peut avoir accès à ces arrièresavoirs et à ces arrière-pensées.

Une certaine critique littéraire tient absolument à nous révéler les «secrets» du texte. Le risque consiste moins, d'ailleurs, à se tenir, avec notre complicité, pour le propriétaire des clés ou des trousseaux de clés qui seront censés vous ouvrir les porte qu à 
«transformer l'œuvre en serrure». selon le mot de Julien Gracq. Une certaine psychanalyse (qualifiée aussi de «psychologie des profondeurs»), qui excelle à détecter le sens caché (contenu latent) dissimulé derrière le sens apparent (contenu manifeste), se fait fort de miner ces dites apparences et de débusquer ce qu'elle appelle des "souvenirs écrans». Mais écrans de quoi ? Une certaine sociologie entend nous démontrer que derrière les «pratiques» des acteurs sociaux, il y a toujours des stratégies qui échappent à ces derniers et expliquent en dernière instance leurs manigances. On n'en finirait pas de dresser la liste de tous ceux qui aujourd'hui partent à la recherche de ce qu'à Port-Royal déjà on appelait le "Dieu caché". Mais la palme du dogmatisme et de l'orgueil revient incontestablement à René Girard, qui a trouvé le moyen de nous refiler dans un seul titre de ses livres du «caché» et de la "fondation». Je ne résiste pas au plaisir de rappeler le titre complet. qui annonce en toute modestie Des choses cachées depuis la fondation du monde. Contre cette conception asymétrique du savoir conçue à la manière d'un appareil détecteur de mensonge (le psychanalyste sait ce que son client ignore, le sociologue est là pour dire la vérité des phénomènes sociaux, le critique est infiniment plus compétent que l'auteur pour faire parler les textes), contre cette gravité pontifiante aussi, qui a tendance à poser en se faisant solennelle, contre la lourdeur (qui est le revers de l'adhérence à soi et du culte de l'identité), et en particulier la lourdeur didactique, contre la prétention à la profondeur de tous ces observateurs «pénétrants" (!), j’aurais plutôt tendance à privilégier la surface, la légèreté, la mobilité, ainsi que le désordre apparent, dont je ne vois pas pourquoi il aurait moins de réalité que l' «ordre caché». Aristophane. Crébillon. Matisse. Sciascia. Milan Kundera. Italo Calvino. François Truffaut. Mais aussi un certain nombre de recherches effectuées dans le cadre de la science, qui s intéresse chaque jour davantage à la légèreté, à l'impondérable, à l'infiniment petit, à l'extrêmement mobile. Dans un livre récent dont j'ai emprunté le titre à Gombrowicz, j'ai tenté de voir ce que cela donnerait si on se mettait à revaloriser les valeurs de surface, à cesser de vouloir prendre les choses «à la racine», mais au contraire à observer et à tenter d'analyser ce qu'on appellerait aujourd'hui «les terminaux» : le langage de la peau. Car pourquoi faudrait-il toujours qualifier. l'air méprisant, de «platitudes» ce qui n'est pas le propre d'une intelligence ou d'une sensibilité réputée - écoutez bien - "pénétrante», dénigrer l'écorce au profit de la partie cachée du tronc de l'arbre, l'écume au profit du fond de la mer que nous ne voyons pas, le corps au profit de l' "âme" dont l'existence est pourtant plus qu'incertaine ?

VARIETE DES CULTURES ET VARIATION DANS LA LANGUE : LES BOUGIES ALL.UMEES FT LE COMBAT CONTRE LE VENT

Au début, il y a ce que l'on voit. L'évidente étrangeté du réel, saisi au ras des sensations et qui. contrairement à la parole, ne saurait mentir. L'univers ne parle pas. Tout vient au monde avec des mots, des mots qui répondent à d'autres mots, et alors l'univers devient bourdonnant de consonnes et de voyelles. L'espèce humaine est, comme le dit Francis Ponge, une «espèce parolière». Nous naissons et grandissons a l'intérieur du langage ou plutôt d'une langue particulière, une langue qui nous comprend autant que nous la comprenons.

L'ethnographie, c'est-à-dire la description scrupuleuse de ce que nous voyons ct entendons, prend nécessairement «le parti pris des choses». «Parti pris des choses = tenir compte des mots». écrit encore Francis Ponge. Car il n'existe pour un objet aucune possibilité d'existence significative en lui-même indépendamment du locuteur, du scripteur, du chercheur, du badaud. Le moindre phénomène, le plus petit geste de la vie quotidienne se donne tout entier - et se dérobe simultanément - dans la relation qu'il entretient avec 
les mots. Dès lors, il n'est aucune réflexion qui ne soit réflexion sur le langage, sur le rapport entre les mots et les choses, les mots et les mots, les mots et les êtres humains. La recherche (à la fois au sens d'Einstein et de Proust) est une exploration méthodique des ressources de la langue, et ces ressources sont infinies pour exprimer la diversité, elle aussi infinie, de ce que l'on voit, touche, écoute, bref, de toul ce que l'on rencontre, chaque situation appelant, mieux, provoquant, une forme linguistique singulière. Fixez par exemple votre attention sur la première personne qui se présente à nous (une personne qui nous est familière ou inconnue), sur un événement minuscule (le savon qui vous glisse des mains sur la baignoire, le chat Momo qui, vilain patapon, s'apprête à - boum - faire tomber la petite boîte en mica couleur cerise posée sur votre bureau, ce gros nuage qui passe en ce moment au-dessus de vous et qui ressemble à Orson Welles). Vous vous apercevez que vous n’avez jamais vraiment été altentif, que vous n'avez jamais vraiment observé. Prenez la peine de vous attarder, de décrire ce que vous avez vu et le retentissement que cela provoque en vous. Décrire la moindre touffe d'herbe, la plus petite pousse de basilic, c'est un défi d'une difficulté inouiie. Nommer les choses, les situations, les êtres, c'est allumer des petites bougies qui s'éteignent, il y a du vent. C'est un combat permanent contre le vent. C'est un combat aussi contre cette idée curieuse : celle d'une transposition dans une forme (scientifique, littéraire, peu importe pour l'instant) d'une réalité en soi indépendante et préexistante à celui qui écoute, regarde, parle et écrit. On peut bien sûr mettre ça sur le compte de l'ignorance ou de la paresse. J'ai plutôt tendance à penser qu'il s'agit d'une insensibilité à ce que chaque objet, chaque rencontre ont de spécifique et appellent par conséquent chaque fois des formes lexicales et grammaticales particulières. Je suis personnellement frappé par l'extraordinaire indifférence d'une grande partie des chercheurs en sciences humaines pour les mots, c'est-àdire le peu d'étonnement qu'ils manifestent devant les potentialités du langage. Le texte «scientifique», écrit comme c'est souvent le cas non pas à partir, mais en dépit des mots, est approximatif et confus. Les formulations adoptées, inappropriées et interchangeables, ont alors quelque chose de désespéré. Je pense à tous ces "concepts» en fonte que l'on tient à portée de main. à cet outillage clouté, utilisé sans doute par peur de ne pas déraper. et auquel on fait indistinctement appel, quelle que soit la situation à laquelle on est confronté, comme si on voulait ridiculiser ce dont on cherche pourtant à rendre compte.

La pensée est inséparable de son mode d'expression, et les problèmes «de forme» sont en fait des problèmes ade fond». Rien ne peut être écrit d'exact (l'écrit étant un combat permanent contre le conformisme de la parole usée) si les expressions choisies, mais aussi inventées, ne sont pas au moins égales à la densité des phénomènes que l'on se propose d'étudier. Écrire exige une sensibilité à l'irréductibilité des choses, des êtres, des situations et des mots ou plutôt de la rencontre entre ces derniers, qui peut être donnée. mais aussi imaginée, expérimentée.

Si je cherche par exemple à rendre compte des déclarations de ce potentat qui domine orgueilleusement ses sujets en déversant sur eux quotidiennement des propos grandiloquents, je dois introduire de la vanité dans le texte. Je ne pourrai pas avoir recours à de tout petits houts de mots. à de minuscules interjections placées à la fin des phrases, à des virgules si discrètes, tapies au détour d'une ligne, si petites, si petites qu'on les remarque à peine. Inversement, je croise presque chaque matin en allant faire les courses un tout petit chien gringalet attaché à la poignée de la porte d'entrée d'un commerçant, et ce tout petit chien qui tremblote en attendant son maitre. il me regarde avec un regard si triste ! Je me dois cette fois d'introduire de la résignation et de la modestie dans la langue. La ligne des mots doit aussi tenter d'exprimer au plus près la ligne de courbure du petit chien assis. Voici maintenant un automobiliste qui piaffe d'impatience parce qu'il est pris dans un embouteillage. Cerné de toutes parts par tant de carrosseries 
qui convergent les unes vers les autres, abruti en cette fin de joumée par les fumées grises et noires des pots d'échappement et par les bruits des moteurs, il en veut au monde entier et plus particulièrement à ses voisins, mais aussi au gouvernement à l'égard duquel il déverse en ce moment, dans un flot d'injures, toute sa hargne. De toute évidence, son sentiment de colère et d'exaspération contre la race humaine ne pourra trouver sá résolution dans une phrase qui s'étend, s’étire, se détend dans le calme olympien des mots assemblés.

Ce que je veux dire. c'est que la lame tranchante du couteau bien affûté doit couper le lecteur, que le mot feu doit enflammer la page, que l'eau doit l'inonder à travers une phonétique aquatique de mots comme rivière, ruisseau, rigole, grenouille ou encore par la répétition allitérante de la labiale $b$ (que l'on trouve dans bain, baignoire, breuvage. brasserie, bistrot, buvette, bavette, boisson, bouteille, bouillon, bouillotte, bouillie, biberon, imbiber, barboter...) qui constitueront le courant textuel du sens de phrases devenues liquides. Le monde et sa compréhension - nul n'est forcé de s'y livrer, on peut très bien partir à la pêche aux moules - ne tolèrent pas l'indifférence et l'indifférenciation. Mais ils exigent de la précision et de la passion. Il y a un héroïsme de l'écriture, et pour rendre compte par exemple de toute cette mangeaille qui attend les convives de ce repas bien comme il faul. toul le monde n'a pas la verve impudique d'un Flaubert, pour "rugir» dans son «gueuloir» la vulgarité de la bourgeoisie qui se goinfre. d'un Flaubert énorme, énergumène, tonitruant, s’arrachant les cheveux toute la nuit jusqu’à ce qu’il parvienne enfin à trouver le mot juste.

Voyons maintenant ce qu'il en est de l'écriture du social (sociographie) et de l'écriture des cultures (ethnographie). Lorsque la réalité sociale se transforme. comme c'est tout particulièrement le cas aujourd'hui, le langage est appelé à se transformer, et le chercheur doit alors s'adapter linguistiquement à ce qu'il veut analyser ou, plus précisément, adapter les processus de procréation textuelle aux caractéristiques de ce qu'il observe. Il convient alors d'accorder la langue en partant à la recherche de nouvelles expressions formelles. Ce qui s'impose, c'est une reconsidération lexicologique et aussi grammaticale qui soit à la mesure des mutations dans lesquelles nous sommes engagés.

L'une des crises majeures de notre époque est une crise des représentations. Comment saisir, c'est-à-dire comment nommer l'aléatoire, le fugitif, l'évanescent. l'érosion des contours, bref tant de recomposition en cours, par exemple le sens qui paraît s'être enfui. la déroute de l'histoire qui ne s'oriente plus tout a fait dans la direction prévue? II me paraît urgent d'aborder ces questions d'une autre façon que celles auxquelles nous étions accoutumés, c'est-à-dire notamment d'écrire de manière différente. Nos sociétés qui se présentent comme fragmentaires appellent peut-être, tant la question de la "forme» devient impérative, une écriture fragmentée et inachevée.

Mais le défi devient plus grand encore lorsque nous nous trouvons confrontés à une culture qui n'est pas la nôtre, à une langue dans laquelle nous ne sommes pas nés. À la variété des cultures répond une variation dans la langue, ce qui est différent ne pouvant, me semble-t-il. qu être dit différemment. Or, que voyons-nous ou plutôt que lisons-nous ? Quelque chose de curieux qui donne l'impression que la tâche est tellement harassante que le chercheur s'efforce de trouver le plus vite possible le repos dans des idées générales. L'étranger et l'étrangeté font le plus souvent ployer nos catégories mentales. sociales et verbales mais ne les rompent pas. Comment cela est-il possible ? Comment se fait-il que quelles que soient les bizarreries des situations culturelles et morphologiques auxquelles sont confrontés tous les ethnologues du monde, il se trouve toujours un langage parfaitement adéquat, un stock de mots, toujours les mêmes, qui nous sont fournis, prêts à porter, pour en rendre compte. Bizarre. Cela provoque parfois un de ces effets comiques, tandis qu'à force d'être banalisé, tout devient ordonné et ordinaire. 
EXPÉRIMENTATION : L'ÉCRITURE DE L'AMERIQUE LATINE COMME DÉFI SIMULTANEMENT SCIENTIFIQUE ET IITTERAIRE.

Ce que je vais proposer maintenant ne me paraît pas procéder d'un retournement carnavalesque qui consisterait à faire imploser la composante scientifique de mon identité. Il me semble que dans les limites mêmes de la théorie anthropologique, dont nous examinerons plus loin les rapports qu elle entretient avec la théorie de la traduction, il est possible d'avoir une autre conception et surtout une autre exigence d'une écriture du social et de la culture, qui sont aussi une écriture de la rencontre. Voila treize ans maintenant que je mène des recherches en Amérique latine, et plus particulièrement au Brésil. Or. ces sociétés se manifestent comme une série de débordements sémantiques, un flux qui frappe non pas de plein fouet (ce serait trop simple, trop clair et on pourrait se préparer pour y faire face). mais dé côté. les habitudes scientifiques, grammaticales, syntaxiques, lexicologiques que l'on a acquises en Europe et en Amérique du Nord. Ce sont des brouilleuses de repères, des empêcheuses de tourner en rond. Elles déstabilisent. Elles introduisent des contradictions permanentes dans nos certitudes, et jusque dans les certitudes de nos incertitudes. Elles mélangent tout. Hormis, il est vrai, les pauvres et les riches. Cette exubérance subie et voulue se présente comme un grouillement tellurique, historique. culturel, un coït généralisé, une copulation permanente de rythmes et de signes. Tout dans ces sociétés se bouscule. Et c'est lorsque vous croyez être parvenu à démêler des fỉls terriblement emmêlés qu’ils se nouent à nouveau. se croisent. s'assemblent, puis se défont. Mais pas comme vous l'aviez prévu. On rencontre rarement dans ces pays des éléments purs, arrivés d'Europe, par exemple, que l'on pourrait facilement identifier. Ainsi, il est impossible d'isoler ou de construire des "types» à la manière de Max Weber, de traiter séparément un aspect, de dénouer analytiquement les nœuds de ces imbrications, de ces espaces, de ces confluences. Mais, en même temps, si tout est inextricablement enchevêtré. les eaux mêlées arrivent séparées (comme le rio São Francisco à Penedo ou plus distinctement encore lorsque à Santarém, le rio Tapajós, noir, rejoint l'Amazone, jaunâtre), l'extrême profusion côtoie la privation extrême, tant de formes et d'acteurs sociaux s'excluent. se font la guerre. la ligne des classes suivant en gros la ligne des races.

C'est, je m'en excuse auprès du lecteur, un casse-tête plus compliqué encore. Car non seulement, dans cette mosaique, rien ne tient en place, mais comment reconstituer des poupées gigognes désemboîtées dont l'écrivain brésilien Osman Lins a bien compris que certaines pièces ont été retirées et que vous n'aurez aucune chance de les retrouver un jour?

À force d'être torsadées par tant d’alluvions, ces sociétés sont devenues invertébrées. Transgéographiques, transhistoriques, transculturelles. C'est ce qu'il y a de plus loin de nous. L'Amérique latine chamboule notre train-train. Elle est si opposée à nos habitudes. Les Européens cherchent des points de référence, alors qu'il n'y a plus de centre de gravité. Elle pulvérise notre sens de la concordance des temps, des limites et du juste milieu, le ni trop, ni trop peu des Français dont l'un d'eux disait, on s'en souvient, que «la France veut être gouvernée au centre». Et même lorsque les acteurs appartiennent au même milieu que nous, lorsque ce sont, comme en Argentine, pratiquement les mêmes interprètes qu en Europe. quelle différence dans la mise en scène !

En raison du caractère incongru des rapprochements et des exclusions tant sociales que linguistiques, l'Amérique latine est un monstre sémantique qui se prête soit à des reportages ou des photographies à sensation, soit à une activité qui tient de la correction (morale et grammaticale). La tequila est trop forte pour nous, parbleu : nous sommes tentés de la couper avec de l'eau. I.'horreur est insoutenable: nous avons du mal à l'accepter, c’est pourquoi nous en donnons des versions sucrées, édulcorées. L'Amérique 
latine, tachée de sueur, transpirante dans ses villages brûlés de soleil : nous devons la ventiler. Frigorifiée et claquant des dents, comme dans les Andes, il nous faut la réchauffer. Détrempée, nous nous efforçons de la sécher. Vociférant dans le brouhaha des marchés, nous avons envie de baisser le son. Trop grosse pour nous, énorme : pourquoi ne pas la dégrossir, lui faire faire de l'exercice. Si maigrichonne : faisons-lui ingurgiter des vitamines pour la remplumer un peu. Énigmatique, masquée : démasquons-la. Touffue : passons-la à la débroussailleuse. Folle à lier, avec son cœur gros comme ça qui bat la chamade, ne faudrait-il pas lui administrer des bétabloquants ?

Pour déterminer et d'abord pour distinguer les éléments originels, les boutures, les greffes venues de chez nous où, d'ailleurs, nous avons besoin (nous, Occidentaux d'Europe. d'Amérique du Nord, et aussi d'Amérique latine) d'élaguer afin de voir clair à travers la forêt des métaphores, d'arracher des adverbes, des adjectifs, des subordonnées, des constructions sociales et verbales qui font parfois franchement métèque. De démêler. de déboiser, d'éclaircir, de polir (afin de rendre tout lisse), de rétablir le calme, de corriger le bégaiement.

Il n'est pas facile, à vrai dire, de mettre au pas, de faire défiler dans l'ordre naturel, de haut en bas et de gauche à droite. les hommes et les femmes de ces sociétés qui se conduisent au mépris des règles historiques les plus élémentaires de la signalisation. Néanmoins, nous sommes de vieux routiers, on peut nous faire confiance. Nous disposons de toute une artillerie de moules et de modèles qui nous permettent, quand «trop c'est trop", de mettre le feu aux tendances inacceptables, ainsi que d'une chirurgie pouvant opérer toutes les excroissances. Cela, nous savons le faire. Ranger les plumes dans les plumiers. Effacer le spectacle de ces acrobates, ces mimes, ces funambules, ces cracheurs de feu qui, pour quelques pesos, arrêtent les voitures dans les rues de Mexico. Ou au contraire braquer les projecteurs pour mieux mettre en évidence leur incongruité. On civilise, on maquille ou au contraire on excise. On nettoie, on retouche, on recycle, on retraite, on corrige la grammaire, on l'expurge de ses contradictions, on habille les Indiens avec des blue-jeans ou au contraire on leur ajoute des plumes et on accentue au marqueur les rondeurs de leurs femmes.

Le continent américain est un continent immense fait pour des passions immenses. Et cet excès américain esı propre à impulser une écriture de l'Amérique susceptible de nous propulser en dehors de nous-mêmes. Je suis frappé par le fait que les journaux des voyageurs, les "relations" des missionnaires, les rapports des administrateurs, relèvent autant de l'histoire de la littérature que de l'histoire à proprement parler. Ces soldats, ces aventuriers, ces évangélistes. Bernal Díaz del Castillo, Cabeza de Vaca, Pierre Martyr d'Angleria, Oviedo... et Colomb lui-même, sont des écrivains extraordinaires. Écrivains par vocation? Pas du tout : ils n'étaient pas partis pour ça. Confrontés à un monde nouveau, démesuré, inimaginable pour eux, ils deviennent écrivains. L'observation de ce qui jamais n'avait été vu est une telle aventure qu'elle transforme la Conquête en événement littéraire : écrire ce qui jamais n'avait été écrit. C'est le choc de l'Ámérique, devenue passion américaine, qui les a conduits à inventer des formes nouvelles d'expression. Pourquoi pas nous ? Nous, voyageurs, chercheurs ou lecteurs au sens borgésien du terme.

Dans l'ouvrage que je me suis permis de vous mentionner plus haut', il m'est apparu qu'il n'était guère possible de nommer ce qui est simultanément un tourbillon cosmique et verbal sans un torrent dans l'écriture. Je pense aux phrases interminables de L'Automne du patriarche, qui s'étendent sur près d'une dizaine de pages, aux longues phrases de Carpentier n'introduisant pas de subordination et donnant l'impression qu'elles ne vont jamais s'arrêter. Comment exprimer également l'excentricité du carnaval, l'orgie du football ou de la fièvre du samedi soir, la mort. le sacrifice, bref, ce que Bataille appelle la dépense - qui est l'expérience de l'extrême - sans une écriture de 
l'extrême ? Comment rendre compte de la gratuité et du ludique si le texte n'est pas luimême enjoué (par exemple, farceur comme chez Borges, malicieux comme chez Cortázar) ? Comment dire la forêt touffue, comment désigner la jungle qui devient ville sans une ampleur stylistique (par exemple la densité de Paradiso, de Lezama Lima)? Comment parler de la figuration et du déguisement sans une écriture qui ne s'avancerait pas masquée? Comment évoquer la complexité, la pluralité, la multiplicité, l'hétérogénéité sociale et culturelle sans une multiplicité dans le texte, sans une écriture elle-même hétéroclite? Telles sont quelques-unes des questions auxquelles j'ai été confronté. J'ai par exemple souvent cherché - sans aucune assurance d'y parvenir — à introduire le chaos dans l'écriture afin que le lecteur soit placé dans une situation analogue à celle de l'état chaotique de ces sociétés. Ou encore, pour dire la partie nonoccidentale du Brésil, celle qui depuis cinq siècles n'a cessé de baver sur l'autre, j'ai eu sans cesse à l'esprit l'intuition du peintre Di Cavalcanti qui, le premier, a compris que "le Brésil est une femme». J'ai tenté alors d'arrondir les phrases - dans lesquelles interviennent notamment les afro-brésilien - estimant que le sens ne pouvait être dans ce cas précis suggéré qu’à partir de la rotondité des formes phonétiques et syntaxiques.

Mais quel effort! L'Amérique latine est un cyclone appelant un orage de mots, une exubérance dans l'écriture, un souffle immense dans les phrases, fail pour les plumes de plus haute volée que la mienne. Des plumes qui doivent également se montrer particulièrement pointilleuses. Car si l'inondation de lumière qui vous tombe dessus en rafale vous invite à un langage phosphorescent, l'éclat du soleil risque de vous éblouir. On croit atteindre la perfection, on attrape une congestion. Et puis il $n$ 'y a pas que cette vaste saoulerie, ce bordel généralisé. Le plus compliqué est de capter toutes ces nuances, qui vont du hoquet à la convulsion. Impossible d y parvenir sans constituer toute une batterie rythmique. Il y a enfin la leçon de ce que j'appellerai l'écriture minimale, l'équivalent, en quelque sorte. de la peinture minimale dans l'écriture: Borges, Bioy Casares, Silvina Ocampo, mais aussi Rulfo, qui a inventé cette prose sobre, dépouillée, millimétrique qui a été pour moi un exemple chaque fois que j’étais confronté a l'extrême de la misère et de la pénurie des favelas.

\section{L'ILLUSION SEMANTIQUE : ECARTS, ESPACES, INTERSTICES}

Si l'on ne peut exprimer un sens sans une connivence avec un son, autrement dit si seule une substance phonique (phonique, graphique, chromatique) est à même de déclencher le pouvoir évocateur des images, l'expression choisie ou inventée n'en est jamais pour autant adéquate à ce qu elle cherche a exprimer. Le mot n'est pas un doublet de la chose ou d'un autre mot, les langues sont irréductibles les unes aux autres et nous touchons ici à ce que Gérard Genetle appelle l' "l'illusion sémantique», illusion d'une relation qui serait pour ainsi dire naturelle (Cratyle, d'après Platon, mais aussi Flaubert) entre le référent el son expression, illusion d'une homologie, voire d'une adéquation. entre les formes que nous «produisons» et les rapports linguistiques ou sociaux que nous étudions.

La question ici posée est celle de la distance, et notamment de la distance que l'on peut introduire par l'humour et l'ironie, qui sont les deux pôles opposées de la mise en question. Par exemple, la langue inventée par Lautréamont dans les Póssies contredit le discours moral du recueil, et dans Les Chants de Maldoror, le contraste devient encore plus saisissant entre la noirceur de l'objet représenté et l'éclat de l'expression adoptée. Frédéric Chambert, qui est l'un des traducteurs d'Horacio Quiroga, estime que ce qui constitue l'une des particularités du style de l'auteur des Contes d'amour, de folie et de mort, c'est notamment «une suradjectivation délibérée à la mesure de la médiocrité des 
personnages décrits". Un demier exemple nous sera donné par l'écriture d’Onetti. Romancier du pessimisme, du désespoir et de la déprime, beaucoup plus cafardeux encore que Sartre dans La nausée. il décrit des situations horribles et des êtres minables, traînant leur misère dans les bas-fonds et évoluant dans une société féroce et corrompue. mais sous sa plume, cet univers resplendit d'une étonnante beauté. Ainsi donc, les bobos d'un enfant ne doivent pas nécessairement s'accompagner de chialements dans le texte, et si, le sourire aux lèvres, un personnage lève une géraldine pour aller cueillir du muguet un beau dimanche de printemps, une organisation sautillante et primesautière de la phrase ne s'impose pas forcément. Le romancier, le traducteur, l'ethnologue s'efforcent de coïncider avec ce qu'ils cherchent à transmettre. Flaubert écrit : «À force de regarder un caillou, je me suis senti y rentrer"; et Cortázar, qui ne fut pas seulement écrivain, mais traducteur (de l'ceuvre complète d'Edgar Poe en espagnol) cite La lestre du caméléon de Keats : «Si un moineau se pose près de ma fenêtre, je fais partie de son existence et je picore moi aussi.» Poursuivant la métaphore du caméléon, l'auteur de Marelle suggère que traduire, c'est devenir l'autre, en tendant un "pont médiumnique» vers lui.

La traduction permet à ce qui est extérieur de devenir intérieur. Intériorisant la langue de l'autre, elle transforme l'extériorité en expérience intérieure. Néanmoins, le travail de traduction, de même que le travail de l'ethnologie, ne vont jamais jusqu'à l'identification et la fusion. Ils font apparaître, au contraire. des espaces et des interstices sans lesquels $c$ en est fini de la pensée (je veux dire de la pensée non dogmatique). Le vapata que j'ai mangé lors de mon demier séjour à Salvador de Bahia me laisse un goût intact qui ne peut se confondre pour moi avec aucun autre plat. Et il en est de même avec la langue. Au début, c'est un peu comme une pluie de mots qui se déverse sur vous, dont vous identifiez certains, mais pour la plupart desquels vous devez chercher le sens dans le dictionnaire pour reconnaître une montre, les yeux, une olive. un coquillage. un teinturier, une luciole, un médiéviste, un saladier, de l'amertume. Il vous faut apprendre à vivre en portugais. Alors, chaque jour, commencent à sortir de vous des mots nouveaux, mais lentement, en hésitant et parfois en tremblant. Puis les mots en viennent à se former dans la bouche sans que vous ayez à réfléchir. Cependant, jamais le mot escola ne fera surgir en vous votre premier souvenir d'écolier, ni le mot mer les plages brésiliennes. Essayez. donc de chanter une samba en français ou d'interpréter une java en allemand.

Traduire (un texte, un comportement, une émotion), c'est établir des passerelles entre le même et l'autre, traverser des espaces, revenir à soi, repartir sans jamais trouver le repos, parce qu'il n'existe pas de langue universelle, pas d'équivalence parfaite, pas de correspondance aboutie entre les mots et les choses, entre les mots et les mots. La saudade est pratiquement incompréhensible pour les Nord-Américains et pour les Européens (hormis les Portugais qui en font un sentiment national qu ils ont transmis aux Brésiliens), parce qu'elle relie ce qu'ils distinguent : la présence du passé dans le présent qui fait tant de bien, qui fait tant de mal, qui consiste simultanément à souffrir du plaisir passé el à prendre plaisir de la souffrance aujourd hui. Voici quelque chose - je vous invite à y revenir encore, tant cela me semble stimulant - qui décidément n'est pas facile à comprendre pour nous. On est si malheureux d'avoir été heureux, et pourtant si heureux d'être maintenant malheureux, sans pour autant sombrer dans le masochisme. C'est aussi incompréhensible, voyez-vous, que l'Eucharistie. C'est comme l'hostie, cette fois je brûle, j'ai presque trouvé. L'hostie, à certains moments, il y a du sang dedans. À certains autres, il n'y a rien. Et puis, ça dépend des gens. Pour certains, le Christ est présent dans la pâte. Pour d'autres, il n'y est pas.

Et comme l'Eucharistie, la saudade est une relation particulière au temps. C'est un certain mode de relation au temps, mais qui est né de l'espac'e... qui sépare le Portugal du Brésil, et plus généralement l'Europe de l'Amérique. Ce sentiment. bien moins aigu et 
surtout moins cultivé qu'au Brésil, n'en existe pas moins dans le reste de l'Amérique latine, constituée aussi de peuples déportés et transplantés : Africains. Européens el bien sûr Indiens. Il existe une nostalgie indienne du paradis perdu. Il existe une nostalgie africaine de la terre que l'on ne reverra jamais plus. Il existe enfin une nostalgie venue d'Espagne, et que l'on rencontre notamment chez. Góngora, qui fait résonner très fort deux termes magiques qui vont littéralement enthousiasmer les Latino-Américains: gozar (jouir) et soledad (la solitude). La solitude, l'absence qui doit, mais ne peut être comblée, mais dont l'être humain peut apprendre à jouir : nostalgia.

\section{ECRITURE FT ETRANGETE.}

Ainsi que l'écrit Italo Calvino, «la littérature est tout entière implicite dans le langage». Elle est le langage lui-même, dans son aptitude non pas à attraper et à fixer des expériences, mais au contraire à les préciser, par exemple en les déployant comme dans le roman ou en les contractant comme dans la nouvelle. L'ethnographie ou la traduction activité elle aussi translinguistique et transculturelle s'il en est - ne disposent probablement pas en ce qui les concerne de la même liberté. Alors que le romancier peut se demander pourquoi nous naissons toujours comme ça les uns après les autres et non pas tous en même temps. et ne connaît. pour répondre à celte question, que les contraintes qu'il s'est lui-même imposées, le traducteur comme l'ethnographe sont. eux, confrontés à un référent: un texte déjà écrit dans le premier cas, une société déjà existante dans le second, dont il faut bien rendre compte. Si la tentation du premier consiste probablement à vouloir à tout prix "coller au texte", l'inclination du second me semble s'apparenter au néoréalisme. L'écriture littéraire, en ce qui la concerne, est à la recherche d'un référent absent. Écrire ne consiste pas à «exprimer», "représenter», «désigner» un contenu qui préexisterait à l'acte de l'écriture, mais à faire advenir ce qui n'existait pas. La traduction. cependant, ne consiste pas davantage en une reproduction, une redite, une répétition, une réplique, un double du texte premier. Le déjà dit - déjà là - est dit d'une manière inédite, car s'il y a bien une forme initiale du texte liéc à une langue originelle et originale dans laquelle il est écrit, le texte existe aussi désormais dans les différentes manières de le formuler (et de le dire).

$\mathrm{Ce} q u$ 'ont également en commun l'ethnographie, la traduction et la littérature (je veux dire la "littérature intrinsèque» au sens de Paul Valéry, l'écriture considérée comme fin - le langage - ce qui dans la rationalité scientifique est plutôt considéré comme moyen), me semble être un souci d'extrême précision. Il s'agit, dans tous les cas, de saisir les nuances imperceptibles qui séparent une brindille d'une autre brindille, un mot d'un autre mot, de réfléchir au détail et au détail du détail. Ce sont des activités en révolte permanente contre les conventions sociales ou linguistiques du vague, du confus, de l'àpeu-près, ainsi que du statu quo des discours ordinaires. Elles font l'expérience de l'insuffisance tant du langage quotidien dans ce qu'il a de lamentablement stéréotypé et répétitif que d'une certaine écriture "scientifique" qui ne semble pas particulièrement se soucier des mots. L'objet de la littérature, de la traduction et de l'ethnographie. c'est l'autre: l'autre de la langue, l'autre du texte, l'autre de la société. Écrire, traduire, exprimer la spécificité d'une culture dans laquelle je ne suis pas né, c'est cheminer en dehors de soi, c'est devenir autre. C'est effectuer à partir de la rencontre des mots une expérience de l'étrangeté pouvant provoquer un sentiment de jubilation, mais aussi de panique. Alors, le réel ne peut pas être lu et écrit à partir d'une appréhension univoque. L'acte de traduire (un texte, une société, ce ne sont que deux modalités d'un défi simultanément littéraire et scientifique somme toute identique) à travers la découverte du fait que j'étais étranger à moi-même, mais que je ne le savais pas impose pour le moins une 
écriture qui ne bloque pas la pluralité contradictoire des significations auxquelles je me trouve confronté.

C'est une experience des limites qui nous fait renoncer, à mon avis irreversiblement, aux mythologies de l'identité (c'est-à-dire de l'adhérence à soi-même) dont je trouve qu'on nous rabat un peu trop les oreilles ces temps-ci : limite de la traduction de ce que je vois, limite de la transparence de ce que j'écris, limite de l'expression, bref, limite de la connaissance.

La littérature, l'ethnologie, la traduction - dont il est vain de se dissimuler ce qui peut aussi les distinguer - sont d'abord des passions, et cela dans les deux sens du terme. Elles nous font connaître le bonheur absolu, au sens strict l'extase. ce qui signifie sortie de soi, mais aussi l'extrême du désespoir (de ne ajamais y arriver»), l'expérience de la séparation et de l'absence. La littérature, l'ethnologie, la traduction - qui contrairement a la philosophie ne sont pas seulement question, mais passion de la question posée en sa singularite - ne tolèrent pas l'amateurisme. Elles exigent un acte de dessaisissement methodique sans cesse reitere, affine, repris, qui consiste à ne plus tenir pour universel ce qui est particulier: notre identite (culturelle, linguistique, sexuelle) par identification exclusive au meme.

Je m'aperçois au terme de ces réflexions que je viens d'énoncer la seule proposition qui personnellement m'importe: estimer qu'il n'y a plus de centre (de centre de référence, de centre de gravite, de centre au monde, plus d'équivalence, plus de plan fixe. plus de langage unique, encore moins de mesure d'une réalite. d'une époque, d'un texte qui serait la verité des autres), mais plusieurs interprétations passées, présentes, à venir, une multitude de traductions possibles auxquelles il ne sera jamais mis un terme, c'est affirmer que la vie l'emporte sur la mort et aussi la mémoire sur l'oubli.

Note

1. François LAPLANTINE (1994) : Transallantique. Entre Europe et Amériques latines, Paris, Editions Payot. 